\title{
Recent advances in the management of carcinoma of the rectum
}

This article was published in the following Dove Press journal:

Clinical and Experimental Gastroenterology

8 June 2009

Number of times this article has been viewed

\section{Somprakas Basu \\ Vivek Srivastava \\ Vijay K Shukla}

Department of General Surgery, Institute of Medical Sciences, Banaras Hindu University,

Varanasi, India
Correspondence: Somprakas Basu Department of General Surgery, Institute of Medical Sciences, Banaras Hindu University,Varanasi 221005, India

$\mathrm{Tel}+9$ I 979344803 I

Fax +91 5422368163

Email sombasu@hotmail.com
Abstract: In the last two decades rectal cancer has changed from a surgically managed disease into a multidisciplinary treatment model resulting in considerable improvements in the survival and outcome. This has been made possible by better understanding of the tumor biology and oncogenesis, advances in diagnostic and staging investigations, and the changing concepts in surgical excision; from the days of abdominoperineal resection to the concept of "zone of upward spread" and low anterior resection to the era of total mesorectal excision and transanal excision. Efforts are on the way to risk stratification and identification of predictors of nonoperative management. Impressive advances in the adjuvant therapies have seen a sea change in the form of postoperative radiotherapy to preoperative radiotherapy to preoperative chemoradiotherapy and postoperative adjuvant chemotherapy. This multidisciplinary approach is the key to impressive local control rates, decreased metastatic rates, overall survival, and enhancement in quality of life. Newer ideas in the understanding of genetic differences in rectal cancers have stemmed from the observation that these cancers differ in their response to the adjuvant treatment. The present day research has focused these areas of biologic differences in cancers and aims to target the specific loci in malignant cells with monoclonal antibodies directed against various growth factors, key enzyme inhibition, and genetic manipulation. The future research lies in the study of gene expression, micro-array techniques, molecular markers, and better understanding of the predictors of tumor response to therapy.

Keywords: carcinoma of the rectum, recent advances, neoadjuvant therapy, total mesorectal excision, chemoradiation

\section{Introduction}

In the last 25 years rectal cancer has changed from a surgically managed disease into a multidisciplinary treatment model resulting in considerable improvements in the survival and outcome of rectal carcinoma patients. Surgical resection represents the focal point of the multidisciplinary management. Surgery for rectal cancer has ranged from abdominoperineal resection (APR) in first described by Czerny in 1884 to the concept of "zone of upward spread" described by Miles in 1908. ${ }^{1}$ Miles emphasized the importance of performing a wide perineal excision, including removal of the pelvic contents of the rectum, the abdominal attachments of the rectum with a high arterial ligation, and the iliac lymph nodes. However it was William Heald who popularized the total mesorectal excision and revolutionized the surgical treatment of rectal cancer. ${ }^{2}$ This surgical approach to rectal cancer appreciates the subtle fascial planes along with the lymphatic and neural anatomy of the pelvis. He described a "zone of downward spread" within the mesorectum that requires adequate excision in order to reduce local

submit your manuscript | www.dovepress.com 
recurrence. The relationship between tumor location and sphincters and the priority to maintain continence through sphincter preservation adds to the challenge of the present day surgical management of rectal cancer. The choice of surgical approach is dependent upon accurate pretreatment staging as well as the role that chemotherapy and radiation play in the neoadjuvant and adjuvant settings. The evolution of the multidisciplinary management of rectal cancer has resulted in impressive local control rates, overall survival rates, and enhancements in quality of life. Research continues in the laboratory, where significant discoveries and innovations pave the way for future progress.

\section{Epidemiology and etiology}

Colorectal cancer (CRC) is the most common internal malignancy in men and women of western societies and its incidence is rapidly increasing in Asia. Colon cancer affects men and women almost equally, whilst rectal cancer is more common in men (female:male ratio, 1:1.3). ${ }^{3}$ The average lifetime risk for an individual to develop colorectal cancer is approximately $6 \%$. This risk increases two- to fourfold if the patient has a personal history of or a first-degree relative with colorectal cancer. Inflammatory bowel disease (IBD) is another risk factor. In the first 10 years after the initial diagnosis of ulcerative colitis (UC), the incidence of colorectal cancer ranges from 2\%-5\%; however, this risk increases $1 \%$ for each year of disease thereafter. For all patients with UC, the cumulative risk for colorectal cancer at 25 years is $25 \%$. Crohn's colitis is associated with a similar risk for colorectal cancer. This is often not appreciated by clinicians because patients with severe Crohn's disease often undergo proctocolectomy before their long-term risk becomes an issue.

Genetic risk factors also have been implicated in the development of colorectal cancer. One is familial adenomatous polyposis (FAP), an autosomal dominant syndrome with $100 \%$ risk of developing colorectal cancer. The abnormality is caused by a defect in the $A P C$ gene located on chromosome 5q21. Patients with FAP develop hundreds or thousands of adenomas by their twenties, and colorectal cancer develops in all patients by age 50 if untreated. A second genetic abnormality associated with the development of colorectal cancer is related to defects in the mismatch repair genes $M S H 2$ and $M L H 1$. Mismatch repair genes affect the repair of DNA replication errors and spontaneous base repair loss and contribute to hereditary nonpolyposis colorectal cancer (HNPCC). Despite the name, these cancers arise from adenomas and may account for $5 \%$ of all colorectal malignancies.
Dietary fats, especially red-meat fats, have been implicated as risk factors for colon and rectal cancer. ${ }^{4}$ Populations that consume less than $15 \%$ of their diet as fat have a lower incidence of colorectal cancer, whereas people who take in $20 \%$ of their diet as fat, either as unsaturated animal fat or as highly saturated vegetable oils, have an increased risk of cancer.

Dukes had described "adenoma-to-carcinoma sequence," in 1926, which was further advanced and proven on a genetic model by Vogelstein. ${ }^{5}$ The majority of patients with rectal cancer do not have an inherited component; instead, there is an initiating genetic mutation, such as of an oncogene like ras, that leads to abnormal cell growth. Subsequently, mutations resulting in inactivation of tumor suppressor genes, such as p53, allow for progression to cancer. The time course for polyp development and transformation to cancer is thought to be 5-10 years. Most adenomas remain benign; however, histologic type, polyp size, and evidence of dysplasia are associated with transformation. Approximately 75\%-85\% of adenomas are tubular, $8 \%-15 \%$ are tubulovillous, and $5 \%-10 \%$ are villous. Villous histology is associated with an increased risk of cancer development. About $50 \%$ of polyps greater than $2 \mathrm{~cm}$ in diameter harbor areas of carcinoma, whereas only $1 \%$ of polyps less than $1 \mathrm{~cm}$ in diameter show evidence of malignant transformation.

\section{Staging investigations}

Accurate staging has a critical role in the decision making process of patients with rectal cancer. There is an increasing role for imaging in the staging of rectal cancer before beginning therapy. The four most commonly used imaging modalities include transrectal ultrasound (TRUS), computerized tomography (CT), magnetic resonance imaging (MRI), and positron emission tomography (PET). The information offered by these imaging modalities, necessary to select appropriate treatment, include depth of tumor and adjacent organs invasion (T stage), lymph node involvement ( $\mathrm{N}$ stage), and metastatic spread (M stage).

\section{Transrectal ultrasound}

Transrectal ultrasound (TRUS) is the most accurate preoperative staging tool for rectal cancer (Table 1). Several studies have shown that the overall accuracy for T stage is $67 \%$ to $93 \%$ and for $\mathrm{N}$ stage is $48 \%$ to $61 \% .{ }^{6,7}$ Compared with CT scanning, TRUS permits a more accurate characterization of the primary tumor and the status of the perirectal lymph nodes. Localized cancers involving only the mucosa and submucosa usually can be distinguished from tumors that 
Table I Endoscopic ultrasound staging of rectal tumors

\begin{tabular}{ll}
\hline Stage & Description \\
\hline uTI & $\begin{array}{l}\text { Invasion confined to the mucosa and submucosa } \\
\text { uT2 }\end{array}$ \\
& $\begin{array}{l}\text { Penetration of the muscularis propria but not through } \\
\text { to the mesorectal fat }\end{array}$ \\
uT3 & Invasion into the perirectal fat \\
uT4 & Invasion into the adjacent organ \\
uN0 & No enlargement of lymph nodes \\
uNI & Perirectal lymph nodes enlarged \\
\hline
\end{tabular}

penetrate the muscularis propria or extend through the rectal wall into the perirectal fat.

\section{CT scan of abdomen and pelvis}

Abdominal and pelvic CT scans can demonstrate regional tumor extension, lymphatic and distant metastases, and tumor-related complications such as perforation or fistula formation. CT scan has been the part of the standard preoperative staging for rectal cancer. Its accuracy in determining the depth of invasion is less than that of TRUS or endorectal coil MRI (ecMRI). The sensitivity of CT scan for detecting distant metastasis is higher $(75 \%-87 \%)$ than for detecting perirectal nodal involvement $(45 \%-73 \%)$ or the depth of transmural invasion (70\%-82\%). ${ }^{7}$ Metastatic lymph nodes are hypodense, round and more than $1 \mathrm{~cm}$ in size.

\section{MRI}

Both ecMRI and surface coil MRI are becoming more useful in the pretreatment evaluation of patients with rectal cancer. The ecMRI offers some advantages compared with TRUS. It permits a larger field of view, it may be less operator- and technique-dependent, and it allows study of stenotic tumors. ${ }^{8}$ Like TRUS, ecMRI can discriminate small-volume nodal disease and subtle transmural invasion, identify involved perirectal nodes on the basis of characteristics other than size, with accuracy rates of $50 \%-95 \%$. The ecMRI can identify tumor foci not only within the mesorectum but also outside the mesorectal fascia. It has $88 \%$ accuracy in predicting the stage of disease. The specificity and sensitivity of ecMRI with combined intravenous and endorectal contrast material to predict infiltration of the anal sphincter were $100 \%$ and $90 \%$, respectively. Surface coil MRI may be beneficial in predicting tumor-free resection margin by visualizing involvement of mesorectal fascia. A recent meta-analysis of TRUS, CT, and MRI reported that TRUS and MRI had similar sensitivity (94\%) in T staging, but TRUS had higher specificity $(86 \%)$ than MRI $(69 \%) .{ }^{9}$ Of great concern was the equally poor sensitivity (67\%) and specificity (77\%) of TRUS and MRI in detecting lymph node metastases. $\mathrm{CT}$ scan cannot reliably predict $\mathrm{T}$ and $\mathrm{N}$ stage. TRUS is limited by its inability to detect lymph nodes outside the range of its transducer (such as iliac, mesenteric, inguinal, and retroperitoneal). CT and MRI offer the advantage of providing detailed anatomic information, as well as detecting abdominal and pelvic metastatic spread.

\section{PET}

Metabolic imaging with PET has an evolving role in assessing the extent of pathologic response of primary rectal cancer to preoperative chemoradiation and may predict long-term outcome. Additionally, it has an accuracy of $87 \%$ for detecting recurrence of rectal cancer after surgical resection and full-dose external-beam radiation therapy. ${ }^{10}$ While PET scans are positive in $90 \%$ of primary and recurrent tumors and in distant metastases, they are relatively inaccurate for nodal metastases. Two important functions of PET scans are the detection of regional and metastatic disease and the assessment of tumor response to therapy. Recently, several small series have reported that the PET scan has changed the treatment in as many as $17 \%$ of patients and has altered the preoperative stage in nearly $40 \%$ of patients. ${ }^{11}$ The PET scan may be useful in the assessment of tumor response to preoperative therapy and preliminary studies indicate that it is more accurate in assessing response to therapy than the CT scan. ${ }^{12}$ As modern multislice CT scanners can generate images of the chest and abdomen in one breath hold, they have become a very effective tool for screening distant metastases and with a sensitivity varying between $70 \%-85 \%, \mathrm{CT}$ is the second most sensitive noninvasive diagnostic tool for the detection of liver metastases, after contrast-enhanced MRI. ${ }^{13}$ When the functional information of 2-fluoro-2-deoxy-D-glucose (FDG)-PET is added to the CT images in hybrid whole body PET-CT imaging, the sensitivity for lesion detection is further improved. ${ }^{13}$ Whole body MRI will become a serious competitor to PET-CT for distant staging of colorectal cancer patients as MRI is less costly than PET-CT and there is no exposure to radiation. ${ }^{14}$

\section{Preoperative bowel preparation}

The role of mechanical bowel preparation (MBP) in rectal surgeries is still evolving. Since most of the studies involve colonic resections and only few studies have focused only on rectal resections, the body of evidence is inadequate. Traditionally, rectal washouts were common and are still in widely practiced. However in a in a multicenter randomized trial by 
Contant and colleagues, ${ }^{15}$ the rate of anastomotic leakage did not differ significantly between with and without MBP groups $(p<0.69)$. Although other septic complications, fascial dehiscence and mortality did not differ, those with MBP had fewer abscesses after anastomotic leakage ( $p<0.001)$. In a study by Bretagnol and colleagues ${ }^{16}$ in 2007,51 consecutive patients with rectal resection without MBP were compared to a matched group of 61 controls with MBP, which showed an overall higher morbidity in the MBP group. A similar anastomotic leak rate ( $8 \%$ vs $10 \% ; p=1.000)$ was observed, though peritonitis occurred more in the no-MBP group and a trend toward higher infectious complications was noted in the MBP group. Another nonrandomized retrospective study $(n=144)$ from the Netherlands, which included low anterior resections without MBP reported a less than 5\% anastomotic leakage rate as compared to a leakage rate of up to $18 \%$ in studies using MBP. ${ }^{17}$ Based on the accrued evidence it is too premature at present to come to a conclusion whether MBP should be omitted in rectal surgeries. ${ }^{18}$

\section{Conceptual advances in surgical resection of rectal cancer}

The primary goal of surgical treatment for rectal cancer is complete eradication of the primary tumor along with the adjacent mesorectal tissue and the superior rectal artery pedicle. Although the aim of surgery is to maintain bowel continuity, cancer removal should not be compromised in an attempt to avoid a permanent colostomy. After establishing the diagnosis and completing the staging work-up, a decision is made whether to pursue immediate resection or administer preoperative chemoradiotherapy depending on the site, fixity and operability, histological type, grade and stage including intramural extent and lymph node status.

\section{Optimal distal resection margins}

For surgically treated rectal cancer, the optimal distal resection margin remains controversial. Although the first line of rectal cancer spread is upward along the lymphatics, tumors below the peritoneal reflection can spread distally via intraor extramural lymphatic and vascular routes. The use of APR for low rectal cancers traditionally has been based on the need for a 5 -cm distal margin of normal tissue. Distal intramural spread usually is limited to within $2.0 \mathrm{~cm}$ of the tumor unless the lesion is poorly differentiated or widely metastatic. The National Surgical Adjuvant Breast and Bowel Project demonstrated no significant differences in survival or local recurrence when comparing distal rectal margins of less than $2 \mathrm{~cm}, 2-2.9 \mathrm{~cm}$, and greater than $3 \mathrm{~cm} .{ }^{19}$ Therefore, a 2-cm distal margin is acceptable for resection of rectal carcinoma. Now with the use of neoadjuvant chemoradiation and Frozen section biopsy of resected margins, $<1 \mathrm{~cm}$ distal margin is also acceptable for distal rectal cancer to preserve the sphincters. ${ }^{20}$

\section{Optimal radial resection margins}

In the last decade, the importance of obtaining an adequate radial margin has been appreciated and stressed upon. Tumor involvement of the circumferential margin (CRM) has been shown to be an independent predictor of local recurrence, distant metastasis, and overall survival. ${ }^{21}$ In their review of more than 17,500 patients, Nagtegaal and Quirke ${ }^{21}$ demonstrated that following neoadjuvant therapy (both radiotherapy and radiochemotherapy) the predictive value of the CRM for local recurrence is significantly higher than when no preoperative therapy has been applied. So the radial margin is more critical than the proximal or distal margin for local control. In one report of 90 patients undergoing resection for rectal cancer, the length of mesorectum beyond the primary tumor that needs to be removed was thought to be between 3 and $5 \mathrm{~cm}$ because tumor implants usually are seen no further than $4 \mathrm{~cm}$ from the distal edge of the tumor within the mesorectum. ${ }^{22}$ Therefore, in proximal rectal cancer, mesorectal excision $5 \mathrm{~cm}$ below the lower border of the tumor should be the goal.

\section{Total mesorectal excision and autonomic nerve preservation}

Locoregional tumor control in rectal cancer surgery has improved significantly over the last 15 years after the introduction of total mesorectal excision (TME). The concept of total mesorectal excision (TME) proposed by Heald has been shown to improve disease free and overall survival. ${ }^{2}$ In 1986, Quirke and colleagues proved that pelvic recurrence is a direct consequence of inadequate mesorectal excision, which leaves regional disease in the pelvis. ${ }^{23} \mathrm{TME}$ is based on the fact that the mesorectum is often a site of nodal or occult micrometastatic disease that is frequently left in situ within the pelvis, when the mesorectum is bluntly mobilized and divided during "traditional" surgery. TME involves sharp dissection under direct vision in the avascular areolar plane between the fascia propria of the rectum, which encompasses the mesorectum, and the parietal fascia overlying the pelvic wall structures. ${ }^{24}$ This procedure emphasizes autonomic nerve preservation. TME in conjunction with an LAR or APR involves precise dissection and removal of the entire rectal mesentery, including that distal to the tumor, as an intact unit. 
A large international retrospective study reported a local recurrence rate of $32 \%$ to $35 \%$ following conventional surgery compared with $4 \%$ to $9 \%$ following TME. ${ }^{25}$ The study also reported a $30 \%$ absolute increase in the overall survival and cancer-specific survival in the TME group. The Dutch ColoRectal Cancer Group noted similar findings when they analyzed data from two prospective randomized studies. The introduction of TME decreased the local recurrence rate from $16 \%$ to $9 \%$, and TME was an independent predictor of overall survival. ${ }^{26}$

\section{Extended lymphadenectomy}

Analysis of an intergroup trial on rectal cancer has determined that the minimum number of lymph nodes examined to define node status accurately is $14 .^{27}$ The issue of high ligation of the IMA is with regard to where the vascular pedicle is divided. Traditional ligation of the vascular pedicle is performed just distal to the origin of the left colic artery. However, anatomic studies have revealed that as many as 10 lymph nodes could be found between the origin of the left colic vessel and the origin of the IMA..$^{28}$ Therefore, high ligation of the IMA was proposed as a method to improve resection and survival. However, subsequent reports failed to support the superiority of high ligation, and it is not routinely practiced for oncologic reasons..$^{29}$ Low rectal carcinomas tend to develop lateral node metastasis and it was demonstrated previously that lateral node dissection reduce the local recurrence rate and survival,,${ }^{30}$ but at the cost of high rates of morbidity in the form of urinary and sexual dysfunction. ${ }^{31}$ Sexual dysfunction is observed in $75 \%-95 \%$ of all patients and about $40 \%-50 \%$ patients suffer from urinary dysfunction. ${ }^{30}$ Although the urinary function may improve over time, the sexual functional loss has been observed to be permanent. Thus in the absence of improved survival, the price of such significant morbidity is too high to advocate extended lateral lymphadenectomy as a routine procedure. Later on Sugihara and colleagues reported the efficacy of pelvic autonomic nerve preserving (PANP) while performing lateral lymph node dissection. ${ }^{32}$ However, at present with the routine use of and advancement in the protocol and technique of neoadjuvant and adjuvant therapy, there is no role for prophylactic lateral lymph node dissection in the treatment of rectal cancer.

\section{Local excision}

For selected T1 and T2 lesions without evidence of nodal disease, transanal full-thickness excision often provides an adequate resection of the primary tumor mass and can spare the patient the morbidity of a more extensive rectal resection. However, it does not stage the nodal drainage areas and therefore cannot provide as complete staging and management of the tumor as a definitive resection. After local excision, if the pathology is unfavorable, the patient should be counseled to have further therapy, including chemoradiation therapy and either a low anterior resection or APR with total mesorectal excision. Preoperative staging, primarily with TRUS or MRI, is the most helpful in identifying appropriate patients for a local excision. The present day accepted criteria for tumors amenable to local excision includes a T1 or T2 tumor with a favorable histology. The tumor should not be fixed or large in size ( $>4 \mathrm{~cm})$, and should not occupy more than $40 \%$ of the circumference. Local lymph nodal involement should deter one from local resection..$^{33}$

Tumors less than $3 \mathrm{~cm}$ from the dentate line but not invading the sphincters usually can be resected via a transanal procedure. Tumors $5 \mathrm{~cm}$ from the dentate line may need a transcoccygeal approach or transanal endoscopic microsurgery (TEM). Tumors $7-10 \mathrm{~cm}$ from the dentate line require TEM or should be considered for a low anterior resection.

If pathologic evaluation of the resected specimen indicates high grade, positive margins, lymphovascular or perineural invasion, a more radical surgery is recommended. Local excision for early (T1) rectal cancers is highly attractive because of its low morbidity, rapid recovery time and outstanding long-term functional results. Unfortunately there are no randomized trials addressing this question. The largest published series of local excision alone for $\mathrm{T} 1$ rectal cancer reports tumor recurrence rates of $4 \%$ to $29 \%$ and overall survival rates of $83 \%$ to $100 \% .{ }^{34}$ The majority of tumor recurrences are local. This indicates that inadequate resection of loco-regional disease is a major cause of treatment failure. Once tumor recurrence has been detected, salvage therapy has limited curative potential. Stringency of patient selection is undoubtedly important especially in $\mathrm{T} 2$ tumors.

\section{Laparoscopic rectal resections}

While randomized trials have established laparoscopic colectomy as an accepted procedure for colon cancer, only limited randomized data are available for patients with rectal cancer. The only large randomized trial that has included patients with rectal cancer is the English CLASICC trial, comparing the conventional versus laparoscopic assisted surgery in colorectal cancer. ${ }^{35}$ The trial showed equivalent length of bowel resection, equivalent lymph node harvest, and equivalent complication rates. A significant concern is the high rate of positive surgical margins among all patients, 
and in particular in those patients treated by laparoscopic low anterior resection (12\%) compared with open low anterior resection $(6 \%)$. The study was unable to document a significant postoperative quality of life benefit associated with laparoscopic surgery. Laparoscopic resection for rectal cancer has technical limitations and a potential for harm. Additional randomized trials are needed to document safety, efficacy, and benefit to patients.

\section{Rectal cancer associated with risk factors}

If rectal cancer is associated with ulcerative colitis and familial adenomatous polyposis, the total proctocolectomy is the surgical recommendation. For HNPCC there is a better prognosis with these cancers than when compared with age-matched controls with non-HNPCC colorectal cancer. Surgical recommendation is the standard resection as in other cases of rectal cancer. There is $45 \%$ risk of having metachronous colon cancer, so total colectomy may be justified. The role of panhysterectomy (in women) in same sitting is investigational and has some justification as there is risk of endometrial and ovarian cancer.

\section{Risk of recurrence after resection of rectal cancer}

After surgical resection, a proportion of those treated will relapse, predominantly with distant metastatic disease. Fifty to $60 \%$ of persons with Stage III and $25 \%$ with Stage II disease will relapse within 5 years. ${ }^{36}$ This is due to the presence of micrometastatic disease at the time of surgery. Such disease can potentially be eradicated with the use of adjuvant therapies. The risk of relapse may be estimated by assessing the clinical and histologic features of the cancer. Poor risk clinical features include higher TNM stage, elevated preoperative carcinoembryonic antigen (CEA), insufficient lymph node sampling ( $<10$ nodes), and presentation with colonic perforation or obstruction. ${ }^{37}$ Metastasis to regional lymph nodes is the factor most strongly predictive of outcome following complete surgical resection. Other factors have consistently been implicated as of prognostic value, but remain to be validated in prospective trials. These include the histological grade of the cancer, lymphovascular invasion, residual tumor following neo-adjuvant therapy for rectal cancer, and microsatellite instability (MSI). ${ }^{37}$ There is no prospective evidence to suggest these factors aid assessment of disease-free and overall survival, or serve as predictive factors for adjuvant therapy benefit. Evidence suggests that cancers displaying MSI have a better prognosis stage for stage compared to microsatellite stable cancers and that persons with such cancer may not benefit from adjuvant 5-FU chemotherapy; however, the literature regarding the latter remains conflicting. ${ }^{38,39}$

\section{Advances in adjuvant chemoradiation}

Treatment of rectal cancer aims at improvement in local control and long-term survival with preservation of anal sphincter, bladder, and sexual function while maintaining or improving quality of life. A consistent challenge has centered not only on these important goals but also on the accurate reporting of surgical technique and precise pathologic staging. Critical surgical management issues include obtaining a total mesorectal excision, autonomic nerve and sphincter preservation, circumferential and distal resection margin clearance, restoration of bowel continuity, and enhancement of postoperative quality of life. ${ }^{40}$ Since 1990 postoperative chemoradiation has been the standard of care in localized rectal cancers. The concept of preoperative neoadjuvant chemoradiation though less than a decade old, is a formidable one. The GI Intergroup 0114, a phase III postoperative adjuvant chemoradiation trial including nearly 1,700 patients with stage II and III rectal cancer, which evaluates chemotherapy and chemoradiation with bolus 5-FU with or without leucovorin and/or levamisole, noted significant survival differences determined by the number of lymph nodes resected and examined. ${ }^{41}$ In patients who had 14 or more lymph nodes sampled, survival was $82 \%$, compared with only $68 \%$ in those with 0 to 4 resected nodes. This trial also defined two risk groups based on $\mathrm{T}$ and $\mathrm{N}$ staging. Seven-year survival and local recurrence were significantly more favorable in patients with low-risk $\left(\mathrm{T}_{1-2} \mathrm{~N}_{+}\right.$ or $\left.\mathrm{T}_{3} \mathrm{~N}_{0}\right)$ disease than those with high-risk $\left(\mathrm{T}_{3} \mathrm{~N}_{+}\right.$or $\left.\mathrm{T}_{4} \mathrm{Nany}\right)$ disease $(70 \%$ versus $45 \%$, respectively [p $<0.0001] ; 9 \%$ versus $18 \%$, respectively [ $p<0.0001])$.

Of note, neither of the two most recent postoperative GI Intergroup trials evaluating chemotherapy and chemoradiation (INT 0114 and INT 0144) has shown a relapse-free or overall survival advantage favoring any 5-FU schedule, whether administered as bolus or infusion therapy or administered with leucovorin and/or levamisole. ${ }^{41,42}$ However, the original work on this topic by O'Connell and colleagues ${ }^{43}$ demonstrating a $10 \%$ benefit of protracted venous infusion of 5 -FU over the bolus dose in decreasing local tumor relapse ( $47 \%$ to $37 \% ; \mathrm{p}=0.01$ ) and distant metastasis $(40 \%$ to $31 \%$; $\mathrm{p}=0.03)$ still remains a valid practice. Both these techniques have been accepted for concurrent chemoradiotherapy by the National Comprehensive Cancer Network (NCCN) 
clinical guidelines (2009) on management of rectal cancer. Preoperative chemoradiation in patients with stage II and III rectal cancer has clearly emerged as the preferred approach, with goals of improving the number of patients obtaining a complete response and decreased local recurrence rate, increasing both the resectability rate and the ability to perform sphincter-sparing surgery, downstaging based on clinical $\mathrm{T}$ and $\mathrm{N}$ status, and reducing the morbidity of combined modality therapy. As one example, the German Rectal Cancer Group compared neoadjuvant versus adjuvant chemotherapy and radiation, showing improved local recurrence $(6 \%$ versus $13 \% ; p=0.0006)$ and twice the number of sphincter-sparing procedures with the neoadjuvant approach; however, five-year disease-free and overall survival were comparable. ${ }^{44}$ NASBP R-03 data from the 253 patients suggested no significant trend toward improvement at three years favoring preoperative versus postoperative therapy (overall survival, $85 \%$ versus $78 \%$, respectively $[p=0.15]$; disease-free survival, $70 \%$ versus $65 \%$, respectively [ $\mathrm{p}=0.40]$; and relapse-free survival, $77 \%$ versus $70 \%$, respectively $[\mathrm{p}=0.22]) .{ }^{45}$

Since both preoperative and postoperative adjuvant strategies were used in the United States, the GI Intergroup designed E3201 in 2001 for comparison of two strategies. The trial offered a "dealers' choice," allowing patients and physicians to select either preoperative chemotherapy and radiation or postoperative combined modality therapy. Because both irinotecan and oxaliplatin at the time were incorporated in adjuvant colon cancer clinical trials, E3201 also planned to compare the effectiveness of combination therapy in a three-arm design evaluating FOLFOX versus FOLFIRI versus 5-FU/LV. Subsequently E3201 was terminated due to a growing preference for preoperative chemoradiation and an interest in biological treatment combinations. Followup data in 123 patients provided important comparative toxicity information, showing that FOLFOX can be safely administered to patients with rectal cancer following chemoradiation. ${ }^{46}$

Recent phase I/II neoadjuvant rectal cancer trials have focused on the role of combination chemotherapy regimens with radiation as an effort to further improve complete response before surgical resection. CALGB 89901 evaluated continuous infusion 5-FU, weekly oxaliplatin, and radiation in patients with $\mathrm{T} 3$ or $\mathrm{T} 4$ rectal cancer showing a complete pathologic response of $25 \%{ }^{47}$ Eastern Cooperative Oncology Group designed a phase I oxaliplatin dose escalation trial, administering every other week oxaliplatin with continuous infusion 5-FU and radiation; the rate of microscopic residual disease was found to be $50 \%$, with a pathologic complete response of $30 \% .{ }^{48}$ Preoperative radiation, oxaliplatin and capecitabine resulted in significant downstaging of patients in a European trial. ${ }^{49}$ In addition, a small phase I rectal cancer trial suggested an antivascular and antitumor effect with the addition of the monoclonal antibody bevacizumab. ${ }^{50}$

The adverse effect of preoperative radiotherapy remains an area of concern in rectal cancer patients as radiation toxicity adversely affects the quality of life. Acute toxicity includes complications of wound healing especially perineal wound healing, gastrointestinal complaints like nausea, vomiting, intestinal obstruction, enteritis and proctitis, genitourinary and neurological symptoms, while late adverse effects occur in the skin, urinary tract, vascular and the skeletal system. The magnitude of morbidity has not been thoroughly documented as few studies have analyzed the outcome after long-term follow up. In a large series, Birgisson and colleagues ${ }^{51}$ compared the adverse outcome of preoperative radiotherapy followed by surgery to surgery alone and found that irradiated patients had higher readmission rates, increased incidence of gastrointestinal symptoms like nausea, abdominal pain, and importantly, bowel obstruction.

Numerous rectal cancer treatment questions remain unanswered. Are there subsets of patients who may not require radiation therapy? Gunderson and colleagues ${ }^{52}$ have evaluated the effect of $\mathrm{T}$ and $\mathrm{N}$ stage and treatment on survival and relapse of rectal cancer as a pooled analysis. The authors observed that patients with intermediate-risk rectal cancer $\left(\mathrm{T}_{1,2} \mathrm{~N}_{1}, \mathrm{~T}_{3} \mathrm{~N}_{0}\right)$ seem to derive no additional benefit from radiation in combination with surgery and chemotherapy when compared to patients treated with surgery and chemotherapy without radiation. In Europe, investigators have studied short course radiation schedules, which, in contrast to prolonged course radiation, raises questions about the importance of downstaging and surgical resectability as well as the effect of pathologic complete response on survival. ${ }^{53,54}$ The second important question raises the issue whether in selected patients with rectal carcinoma receiving chemoradiotherapy (CRT), surgery may be spared. Pathologic complete response rate of $25 \%-30 \%$ have been observed in early stage rectal tumors after completion of CRT. ${ }^{55,56}$ This questions the role of surgery. In a prospective study by Habr-Gama and colleagues,${ }^{57}$ the patients with $\mathrm{T} 2-\mathrm{T} 4$ tumors received 5FU-based CRT preoperatively. After eight weeks, they were reassessed and 71 out of 265 patients $(26.8 \%)$ had complete clinical response, and were spared of surgery 
and followed up, while the rest were treated with rectal resection. The five-year overall survival rates were similar, $88 \%$ in the observed group compared to $92 \%$ in the surgery group (not significant). In another study by Onaitis and colleagues, observation showed no significant advantage in local recurrence, disease-free survival and overall survival rates between the clinically complete and partial responders and pathologically complete and partial responders. ${ }^{58}$ The authors concluded that clinical evaluation following neoadjuvant CRT is unreliable as micrometastasis may persist. Hence observation policy in these patients should be taken with caution. Although in a subset of patient surgery may be spared after neoadjuvant CRT, it is unclear at present which patients are going to respond completely and thus prediction is difficult and not backed by sound evidence. Bigger prospective series are required to identify the factors responsible for clinical and pathological response, especially complete response in rectal cancers. It is thus believed that the best opportunity to improve survival in patients with rectal cancer will require continued focus on adjuvant chemoradiotherapy strategies, while at the same time pursuing detailed assessment of both acute and chronic toxicities. Additionally, if treatment strategies are to be successful in patients with rectal cancer, enhanced understanding of tumor biology is essential.

\section{Other advances in treatment of rectal carcinoma Immobilization molds and tissue expanders}

A number of investigators have evaluated the effectiveness of custom bowel immobilization molds and other devices to decrease the amount of small bowel irradiated. ${ }^{59}$ These have generally been partially effective in minimizing bowel irradiation, but are dependent on precise location of the device relative to the patient. For patients treated postoperatively, surgical maneuvers to keep small bowel from being fixed in the pelvis can be effective in minimizing bowel irradiation. Pelvic tissue expanders have been used to decrease in small bowel volume in the radiation field and a decrease in acute toxicity. Reperitonealizing the pelvic floor or having a surgical flap inserted into the pelvis can also be of substantial use, and these are the techniques most commonly used at present. The placement of surgical clips in the high-risk areas in the pelvis to better define the tumor volume for postoperative irradiation can be of enormous benefit to the radiation oncologist in limiting the extent of the high-dose region.

\section{Monoclonal antibodies and targeted therapy}

Though chemotherapeutic agents form the cornerstone of adjuvant treatment in rectal cancers, development of monoclonal antibodies and rapid incorporation of novel 'targeted' therapies have taken place in the last decade, supported by the results of randomized clinical trials. Among the most promising of these agents are agents that inhibit farnesyl transferase, vascular endothelial growth factor (VEGF) receptor and epidermal growth factor receptor (EGFR), and other inhibitors of angiogenesis. Ras oncogenes are mutated in more than $40 \%$ of colorectal cancers. ${ }^{60}$ Farnesyl transferase facilitates the association of ras through the inner surface of plasma membrane. Inhibition of this enzyme has shown promising antitumor activity and a number of inhibitors of farnesyl transferase have been shown to have significant clinical efficacy. ${ }^{61,62}$ VEGF is another important target since it is a promoter of angiogenesis ${ }^{63}$ and increased expression of VEGF is associated with increased metastasis and poor prognosis in colorectal cancer. ${ }^{64}$ Several strategies for inhibition of VEGF like VEGF antisense, monoclonal antibodies and specific small molecule inhibitors have been developed. ${ }^{65}$ Bevacizumab, the monoclonal antibody targeting VEGF, as well as cetuximab and panitumumab, which are monoclonal antibodies against EGFR, have recently been approved to be used against advanced colorectal cancer. Although bevacizumab seems to have no significant clinical activity as a single agent in advanced colorectal cancer and the activity of single-agent cetuximab is only modest, their efficacy improves when combined with chemotherapy. ${ }^{66,67}$ Cetuximab has been shown to increase the efficacy of irinotecan in irinotecan-refractory patients, indicating that cetuximab may make tumors more sensitive to chemotherapeutic agents. ${ }^{68}$ Furthermore, a combined analysis of three studies suggests that the addition of bevacizumab may even compensate for the lack of an active chemotherapeutic agent in the first-line setting because patients treated with FU/leucovorin plus bevacizumab had improved results when compared with patients administered FU/ leucovorin or irinotecan/FU/leucovorin. ${ }^{69}$ Moreover, novel antibodies and tyrosine kinase inhibitors are undergoing active investigation in clinical trials. ${ }^{70}$

\section{Dietary supplements and radioprotectors}

Randomized trials have examined the efficacy of various compounds to decrease bowel toxicity. These trials have included such compounds as butyric acid to decrease chronic radiation proctitis, sucralfate enemas to decrease acute 
radiation proctitis, olsalazine to decrease acute enteritis, and mesalazine (5-aminosalicylic acid) to decrease acute radiation enteritis. ${ }^{71}$ All of these trials have been negative. A lactose-restricted diet has not been effective in decreasing symptoms, and the radioprotector WR-2721 also has not shown benefit in some trials but with a suggestion of benefit in others.

\section{Molecular predictive markers}

With better understanding of the tumor biology and molecular phenotype of rectal cancers, several biological markers have shown to be useful predictors of outcome and/or response to treatment. The availability of a number of active agents has helped select a particular drug or a drug combination that would have an increased likelihood of efficacy or a decreased likelihood of toxicity. Of these the most extensively studied are thymidylate synthase (TS) and $p 53$. It has been seen that TS activity correlates directly with response to $5-\mathrm{FU}$ in advanced disease ${ }^{72}$ and also as predictor of survival and disease-free survival. ${ }^{73}$ Although the prognostic significance of $p 53$ is not clear, an interaction between $p 53$ and TS levels has been observed. ${ }^{74}$ Another promising avenue of investigation has been the elucidation of markers of resistance to 5-FU-based chemotherapy derived from the knowledge of its metabolic pathways. It has been observed that high levels of dihydropyrimidine dehydrogenase (DPD) or thymidine phosphorylase (TP), as measured in a tumor specimen by reverse transcriptase-polymerase chain reaction, predict a failure to respond to an infusional 5-FU regimen.

Allelic loss is widespread in colorectal cancers and the clinical significance of allele loss of $17 p$ and $18 q$ has been defined in predicting prognosis ${ }^{75,76}$ along with MSI and transforming growth factor $\beta-1$ receptor II mutation. Patients without $18 \mathrm{q}$ loss of heterozygosity (LOH) and with MSI positive tumors with transforming growth factor $\beta$-1 receptor II mutation have the best prognosis. ${ }^{77}$ MSI-positive patients with colorectal cancers respond significantly better to adjuvant 5-FU/levamisole chemotherapy. ${ }^{78}$

\section{Gene therapy and gene expression}

Colorectal cancer is a reasonable potential target for gene therapy approaches because regional administration of a gene vector may be practical. Trials of different gene therapy approaches, including virus-directed enzyme prodrug therapy, immunogenic manipulation, gene correction, and viral therapy have all been initiated. ${ }^{79,80}$
One of the most important advances in rectal cancer management has been the development of tumor banks. ${ }^{81}$ The gene signatures from DNA chip analysis (DNA microarray) are already in use in predicting the response to adjuvant therapy, defining the low and high risk groups and predicting distant metastasis. These gene-expression techniques have also been used to predict response to radiotherapy with an accuracy as high as $82.4 \% .^{82}$ Similar results have been reported by Ghadimi and colleagues ${ }^{83}$ when the gene expression was evaluated on a small subset of patients from the German Rectal Cancer Trial (CAO/ARO/AIO-94). The authors studied the expression of 54 genes in 23 patients with locally advanced rectal cancer and demonstrated positive and negative predictive values of $78 \%$ and $86 \%$, respectively, in detecting the probable responders to preoperative chemoradiotherapy.

\section{Conclusion}

The recent trend of events in management of rectal cancer indicates a multimodality approach. Although sphincter preservation gives a better quality of life, it should not be the aim at the cost of curative resection. Early rectal cancers are favorable tumors and specific subsets are amenable to local excision. The role of transanal endoscopic microsurgery is promising in this regard. With evolution of recent imaging modalities like TRUS, MRI, PET-CT scans, etc., the exact stage determination is possible, which is an important step in the work up of a patient with rectal cancer. Treatment of locally advanced disease has been the most explored area in the last decade. In brief, progression from postoperative RT to preoperative RT to preoperative CRT to improved chemotherapy techniques (drugs increasing effect of chemotherapy) in preoperative CRT to addition of adjuvant postoperative CT has best defined the changing trends in the treatment of rectal cancer with an aim to eradicate local recurrence and in the long run to increase survival. The concurrent concepts of short and long-course preoperative RT although still present, are giving way to neoadjuvant CRT. Although neoadjuvant CRT decreases local recurrence, it does not improve survival. All these advances have been made possible by better understanding of tumor biology especially various gene expression of rectal cancers. Predictive markers of cancer response to CRT are very much needed not only to decrease useless treatment but also to better select the right subset of patients. Lastly, efforts are on the way to assess the predictors of nonoperative management in early rectal tumors who have shown complete clinical and pathological response after preoperative CRT. 


\section{Disclosure}

The authors report no conflicts of interest in this work.

\section{References}

1. Miles WE. A method of performing abdominoperineal excision for carcinoma of the rectum and the terminal portion of the pelvic colon. Cancer. 1908;2:1812.

2. Heald RJ, Moran BJ, Ryall RD, et al. Rectal cancer: The Basingstoke experience of total mesorectal excision, 1978-1997. Arch Surg. 1998;133:894-899.

3. Nelson RL, Dollear T, Freels S, Persky V. The relation of age, race, and gender to the subsite location of colorectal carcinoma. Cancer. 1997;80:193-197.

4. Enker WE, Kafka NJ, Martz J. Planes of sharp pelvic dissection for primary, locally advanced, or recurrent rectal cancer. Semin Surg Oncol. 2000;18:199-206.

5. Fearon ER, Vogelstein B. A genetic model for colorectal carcinogenesis. Cell. 1990;61:759-767.

6. Katsura Y, Yamada K, Ishizawa T. Endorectal ultrasonography for the assessment of wall invasion and lymph node metastasis in rectal cancer. Dis Colon Rectum. 1992;35:362-369.

7. Rifkin MD, Ehrlich SM, Marks G. Staging of rectal carcinoma: prospective comparison of endorectal US and CT. Radiology. 1989;170:319-322.

8. Orrom WJ, Wong WD, Rothenberger DA, et al. Endorectal ultrasound in the preoperative staging of rectal tumors: A learning experience. Dis Colon Rectum. 1990;33:654-659.

9. Bipat S, Glas AS, Slors FJ, Zwinderman AH, Bossuyt PM, Stoker J. Rectal cancer: local staging and assessment of lymph node involvement with endoluminal US, CT, and MR imaging - a meta-analysis. Radiology. 2004;232:773-783.

10. Guillem JG, Moore HG, Akhurst T, et al. Sequential preoperative fluorodeoxyglucose positron emission tomography assessment of response to preoperative chemoradiation: A means for determining long-term outcomes of rectal cancer. J Am Coll Surg. 2004;199:1-7.

11. Kantorova I, Lipska L, Belohlavek O, Visokai V, Trubac M, Schneiderova M. Routine (18) F-FDG PET preoperative staging of colorectal cancer; comparison with conventional staging and its impact on treatment decision making. J Nucl Med. 2003;44:1784-1788.

12. Chessin DB, Kiran RP, Akhurst T, Guillem JG. The emerging role of $18 \mathrm{~F}$ fluorodeoxyglucose positron emission tomography in the management of primary and recurrent rectal cancer. J Am Coll Surg. 2005;1:948-956.

13. Albrecht T, Hohmann J, Oldenburg A, Skrok J, Wolf KJ. Detection and characterization of liver metastases. Eur Radiol. 2004;14 (Suppl 8): P25-P33.

14. Van Etten B, Van Der Sijp J, Kruyt R, Oudkerk M, van der Holt B, Wiggers T. Ferumoxide-enhanced magnetic resonance imaging techniques in pre-operative assessment for colorectal liver metastases. Eur J Surg Oncol. 2002;28:645-651.

15. Contant CM, Hop WC, van't Sant HP, et al. Mechanical bowel preparation for elective colorectal surgery: a multicenter randomized trial. Lancet. 2007;370(9605):2112-2117.

16. Bretagnol F, Alves A, Ricci A, Valleur P, Panis Y. Rectal cancer surgery without mechanical bowel preparation. Br J Surg. 2007;94(10): 1266-1271.

17. Vlot EA, Zeebregts CJ, Gerritsen JJ, Mulder HJ, Mastboom WJ, Klaase JM. Anterior resection of rectal cancer without bowel preparation and diverting stoma. Surg Today. 2005;35(8):629-633.

18. Basu S, Shukla VK. Mechanical bowel preparation: are we ready for a paradigm shift. Dig Surg. 2008;25:325-328.

19. Wolmark N, Fisher B. An analysis of survival and treatment failure following abdominoperineal and sphincter-saving resection in Dukes' B and C rectal carcinoma: A report of the NSABP clinical trials. National Surgical Adjuvant Breast and Bowel Project. Ann Surg. 1986;204:480-489.
20. Nelson H, Petrelli N, Carlin A, et al. Guidelines 2000 for colon and rectal cancer surgery. J Natl Cancer Inst. 2001;93:583-596.

21. Nagtegaal ID, Quirke P. What is the role for the circumferential margin in the modern treatment of rectal cancer? J Clin Oncol. 2008;26:303-312.

22. Hida J, Yasutomi M, Maruyama T, et al. Lymph node metastases detected in the mesorectum distal to carcinoma of the rectum by the clearing method: Justification of total mesorectal excision. J Am Coll Surg. 1997; 184:584.

23. Quirke P, Durdey P, Dixon MF, Williams NS. Local recurrence of rectal adenocarcinoma due to inadequate surgical resection. Histopathology study of lateral tumor spread and surgical excision. Lancet. 1986;2:996-999.

24. Havenga K, Enker WE, McDermott K, et al. Male and female sexual and urinary function after total mesorectal excision with autonomic nerve preservation for carcinoma of the rectum. J Am Coll Surg. 1996;182:495-502.

25. Heald RJ, Ryall RD. Recurrence and survival after total mesorectal excision for rectal cancer. Lancet. 1986;1:1479-1482.

26. Kapiteijn E, Putter H, van de Velde CJ. Cooperative investigators of the Dutch ColoRectal Cancer Group. Impact of the introduction and training of total mesorectal excision on recurrence and survival in rectal cancer in The Netherlands. Br J Surg. 2002;89:1142-1149.

27. Tepper JE, O'Connell MJ, Niedzwiecki D, et al. Impact of number of nodes retrieved on outcome in patients with rectal cancer. J Clin Oncol. 2001;19:157-163.

28. Grinnel RS. Results of ligation of inferior mesenteric artery at the aorta in resections of carcinoma of the descending and sigmoid colon and rectum. Surg Gynecol Obstet. 1965;120:1031-1036.

29. Pezim ME, Nicholls RJ. Survival after high or low ligation of the inferior mesenteric artery during curative surgery for rectal cancer. Ann Surg. 1984;200:729-733.

30. Moriya Y, Hojo K, Sawada T, Koyama Y. Significance of lateral node dissection for advanced rectal carcinoma at or below the peritoneal reflection. Dis Colon Rectum. 1989;32:307-315.

31. Masui H, Ike H, Yamaguchi $\mathrm{S}$, et al. Male sexual function after autonomic nerve-preserving operation for rectal cancer. Dis Colon Rectum. 1996;39:1140-1145.

32. Sugihara K, Moriya Y, Akasu T, Fujita S. Pelvis autonomic nerve preservation for patients with rectal carcinoma. Cancer. 1996;78:1871-1880.

33. Nastro P, Beral D, Hartley J, Monson JRT. Local excision of rectal cancer: review of literature. Dig Surg. 2005;22:6-15.

34. Madbouly KM, Remzi FH, Erkek BA, et al. Recurrence after transanal excision of T1 rectal cancer: should we be concerned? Dis Colon Rectum. 2005;48:711-719.

35. Guillou PJ, Quirke P, Thorpe H, et al. Short-term endpoints of conventional versus laparascopic-assisted surgery in patients with colorectal cancer (MRC CLASICC trial): multicentre, randomised controlled trial. Lancet. 2005;365:1718-1726.

36. Desch CE, Benson AB 3rd, Somerfield MR, et al. Colorectal cancer surveillance: 2005 update of an American Society of Clinical Oncology practice guideline. J Clin Oncol. 2005;23:8512-8519.

37. Compton CC, Fielding LP, Burgart LJ, et al. Prognostic factors in colorectal cancer. College of American Pathologists Consensus Statement 1999. Arch Pathol Lab Med. 2000;124:979-994.

38. Iacopetta B, Elsaleh H, Zeps N. Microsatellite instability in colon cancer. N Engl J Med. 2003;349:1774-1776.

39. Ribic CM, Sargent DJ, Moore MJ, et al. Tumor microsatellite-instability status as a predictor of benefit from fluorouracil-based adjuvant chemotherapy for colon cancer. N Engl J Med. 2003;349:247-257.

40. Balch GC, DeMeo A, Guillem JG. Modern management of rectal cancer: a 2006 update. World J Gastroenterol. 2006;12:3186-3195.

41. Tepper JE, O'Connell M, Niedzwiecki D, et al. Adjuvant therapy in rectal cancer: analysis of stage, sex, and local control-final report of intergroup 0114. J Clin Oncol. 2002;20:1744-1750.

42. Smalley SR, Benedetti JK, Williamson SK, et al. Phase III trial of fluorouracil-based chemotherapy regimens plus radiotherapy in postoperative adjuvant rectal cancer: GI INT 0144. J Clin Oncol. 2006;24:3542-3547. 
43. O'Connell MJ, Martenson JA, Wieand HS, et al. Improving adjuvant therapy for rectal cancer by combining protracted-infusion fluorouracil with radiation therapy after curative surgery. New Engl J Med. 1994;331:502-507.

44. Sauer R, Becker H, Hohenberger W, et al. Preoperative versus postoperative chemoradiotherapy for rectal cancer. $N \mathrm{Engl} \mathrm{J} \mathrm{Med}$. 2004;351:1731-1740.

45. Roh MS, Colangelo L, Wieand S, et al. Response to preoperative multimodality therapy predicts survival in patients with carcinoma of the rectum [abstract]. J Clin Oncol. 2004;22:3505.

46. Benson AB, Catalano PJ, Meropol NJ, et al. ECOG E3201: Intergroup randomized phase III study of postoperative irinotecan, 5-fluorouracil (FU), leucovorin (LV) (FOLFIRI) vs oxaliplatin, FU/LV (FOLFOX) vs FU/LV for patients (pts) with stage II/III rectal cancer receiving either pre or postoperative radiation (RT)/FU [abstract]. J Clin Oncol. 2006;24:3526.

47. Ryan DP, Niedzwiecki D, Hollis D, et al. Phase I/II study of preoperative oxaliplatin, fluorouracil, and external-beam radiation therapy in patients with locally advanced rectal cancer: Cancer and Leukemia Group B 89901. J Clin Oncol. 2006;24:2557-2562.

48. Landry J, Rosenthal D, Spitz F, et al. ECOG1297: A phase I study of preoperative radiation therapy (RT) with concurrent protracted continuous infusion 5-FU and dose escalating oxaliplatin followed by surgery, adjuvant 5-FU, and leucovorin for locally advanced (T3/4) rectal adenocarcinoma. Int JRadiat Oncol Biol Phys. 2003;57:S179.

49. Rodel C, Grabenbauer GG, Papadopoulos T, et al. Phase I/II trial of capecitabine, oxaliplatin, and radiation for rectal cancer. J Clin Oncol. 2003;21:3098-3104.

50. Willett CG, Boucher Y, di Tomaso E, et al. Direct evidence that the VEGF-specific antibody bevacizumab has antivascular effects in human rectal cancer. Nat Med. 2004;10:145-147.

51. Birgisson H, Pahlman L, Gunnarsson U, Glimelius B. Adverse effects of preoperative radiation therapy for rectal cancer: long term follow up of the Swedish Rectal Cancer Trial. J Clin Oncol. 2005;23: 8697-8705.

52. Gunderson LL, Sargent DJ, Tepper JE, et al. Impact of T and N stage and treatment on survival and relapse in adjuvant rectal cancer: a pooled analysis. J Clin Oncol. 2004;22:1785-1796.

53. Sebag-Montefiore D, Steele R, Quirke P, et al. Routine short course pre-op radiotherapy or selective post-op chemoradiotherapy for resectable rectal cancer? Preliminary results of the MRC CR07 randomized trial [abstract]. J Clin Oncol. 2006;24:3511.

54. Quirke P, Sebag-Montefiore D, Steele R, et al. Local recurrence after rectal cancer resection is strongly related to the plane of surgical dissection and is further reduced by pre-operative short course radiotherapy. Preliminary results of the Medical Research Council (MRC) CR07 trial [abstract]. J Clin Oncol. 2006;24:3512.

55. Rödel C, Martus P, Papadoupolos T, et al. Prognostic significance of tumor regression after preoperative chemoradiotherapy for rectal cancer. J Clin Oncol. 2005;23:8688-8696.

56. Kim C, Anne PR, Michel E, et al. Impact of pre-treatment size and lymph nodes on pathological complete response and survival in a prospective trial of chemoradiation [abstract]. Int J Radiat Oncol Biol Phys. 2005;63(Suppl 164):1034.

57. Habr-Gama A, Perez RO, Nadalin W, et al. Long-term results of preoperative chemoradiation for distal rectal cancer correlation between final stage and survival. J Gastrointest Surg. 2005;9:90-9; discussion, 99-101.

58. Onaitis MW, Noone RB, Fields R, et al. Complete response to neoadjuvant chemoradiation for rectal cancer does not affect survival. Ann Surg Oncol. 2001;8:801-806.

59. Fu YT, Lam JC, Tze JM. Measurement of irradiated small bowel volume in pelvic irradiation and the effect of a bellyboard. Clin Oncol ( $R$ Coll Radiol). 1995;7:188-192.

60. Bollag G, McCormick F. Regulators and effectors of ras proteins. Annu Rev Cell Dev Biol. 1991;7:601-632.
61. Lui M, Bryant MS, Chen J, et al. Antitumor activity of SCH66336, an orally bioavailable tricyclic inhibitor of farnesyl protein transferase, in human tumor xenograft models and wap-ras transgenic mice. Cancer Res. 1998;58:4947-4956.

62. Skrzat S, Angibaud P, Venet M, et al. R115777, a novel imidazole farnesyl transferase inhibitor (FTI) with potent oral antitumor activity. Proc Am Soc Cancer Res. 1998;39:2170a.

63. Brown LF, Berse B, Jackman RW, et al. Expression of vascular permeability factor (vascular endothelial growth factor) and its receptor in adenocarcinomas of the gastrointestinal tract. Cancer Res. 1993;53:4727-4735.

64. Takahashi Y, Kitadai Y, Bucana CD, et al. Expression of vascular endothelial growth factor and its receptor, KDR, correlates with vascularity, metastasis and proliferation of human colon cancer. Cancer Res. 1995;55:3964-3968.

65. Ellis L, Takahashi Y, Liu W, et al. Vascular endothelial growth factor in human colon cancer: biology and therapeutic implications. Oncologist. 2000;5:11-15

66. Venook A, Niedzwiecki D, Hollis D. Phase III study of irinotecan/ 5FU/LV (FOLFIRI) or oxaliplatin/5FU/LV (FOLFOX) \pm cetuximab for patients with untreated metastatic adenocarcinoma of the colon and rectum: CALGB 80203 preliminary results. J Clin Oncol. 2006; 24(suppl 188, abstr 3509):148s.

67. Giantonio BJ, Catalano PJ, Meropol NJ. High-dose bevacizumab improves survival when combined with FOLFOX4 in previously treated advanced colorectal cancer: Results from the Eastern Cooperative Oncology Group (ECOG) study 3200. J Clin Oncol. 2005; 23(suppl 168, abstr 2):1s.

68. Goldberg RM, Hurwitz HI, Fuchs CS. The role of targeted therapy in the treatment of colorectal cancer. Clin Adv Hematol Oncol. 2006; 4(8 Suppl 17):1-10.

69. Kabbinavar FF, Hambleton J, Mass RD, Hurwitz HI, Bergsland E, Sakar S. Combined analysis of efficacy: the addition of bevacizumab to fluorouracil/leucovorin improves survival for patients with metastatic colorectal cancer. J Clin Oncol. 2005;23:3706-3712.

70. Saltz LB, Rubin M, Hochster H, et al. Cetuximab (IMC-C225) plus irinotecan (CPT-11) is active in CPT-11 refractory colorectal cancer that expresses epidermal growth factor receptor. Proc Am Soc Clin Oncol. 2001;20:7a.

71. O'Brien PC, Franklin CI, Dear KB, et al. A phase III double-blind randomized study of rectal sucralfate suspension in the prevention of acute radiation proctitis. Radiother Oncol. 1997;45:117-123.

72. Leichman CG, Danenberg PV, Tan M, et al. Quantitation of intratumoral thymidylate synthase expression predicts for disseminated colorectal cancer response and resistance to protracted infusion fluorouracil and weekly leucovorin. J Clin Oncol. 1997;15: 3223-3229.

73. Yamachika T, Nakanishi H, Inada KL, et al. A new prognostic factor for colorectal carcinoma, thymidylate synthase, and its therapeutic significance. Cancer. 1998;82:70-77.

74. Lenz HJ, Hayashi K, Salonga D, et al. P53 point mutations and thymidylate synthase messenger RNA levels in disseminated colorectal cancers: an analysis of response and survival. Clin Cancer Res. 1998;4:1243-1250.

75. Legget B, Young J, Buttenshaw R, et al. Colorectal carcinomas show frequent allelic loss on the long arm of chromosome 17 with evidence for a specific target region. Br J Cancer. 1995;71:1070-1073.

76. Lanza G, Matteuzzi M, Gafa R, et al. Chromosome 18q allelic loss and prognosis in stage II and III colon cancer. Int J Cancer. 1998;79: 390-395.

77. Watanabe T, Wu TT, Catalano PJ, et al. Molecular predictors of survival after adjuvant chemotherapy for colon cancer. $N$ Engl J Med. 2001;344:1196-1206.

78. Elsaleh H, Joseph DJ, Lacopetta B. Microsatellite instability is a strong molecular predictive marker for sensitivity to adjuvant chemotherapy in Duke's C colorectal carcinoma. Proc Am Soc Clin Oncol. 2000;19:238a. 
79. Palmer DH, Chen MJ, Kerr DJ. Gene therapy for colorectal cancer. Br Med Bull. 2002;64:202-225.

80. Chen Y, Chang KJ, Hwang LH, Chen CN, Tseng SH. Establishment and characterization of a rectal cancer model in mice: application to cytokine gene therapy. Int J Colorectal Dis. 2002;17:388-395.

81. Benson III AB. New approaches to assessing and treating early-stage colon and rectal cancers: cooperative group strategies for assessing optimal approaches in early-stage disease. Clin Cancer Res. 2007; 13(22 suppl):6913s-6918s.
82. Watanabe T, Komuro Y, Kiyomatsu T, et al. Prediction of sensitivity of rectal cancer cells in response to preoperative radiotherapy by DNA microarray analysis of gene expression profiles. Cancer Res. 2006;66:3370-3374.

83. Ghadimi BM, Grade M, Difilippantonio MJ, et al. Effectiveness of gene expression profiling for response prediction of rectal adenocarcinomas to preoperative chemoradiotherapy. J Clin Oncol. 2005;23:1826-1838.

\section{Publish your work in this journal}

Clinical and Experimental Gastroenterology is an international, peerreviewed, open access journal, publishing all aspects of gastroenterology in the clinic and laboratory, including: Pathology, pathophysiology of gastrointestinal disease; Investigation and treatment of gastointestinal disease; Pharmacology of drugs used in the alimentary tract;
Immunology/genetics/genomics related to gastrointestinal disease. This journal is indexed on CAS. The manuscript management system is completely online and includes a very quick and fair peer-review system. Visit http://www.dovepress.com/testimonials.php to read real quotes from published authors.

Submit your manuscript here: http://www.dovepress.com/clinical-and-experimental-gastroenterology-journal 